\begin{tabular}{|c|l|}
\hline Title & Boltzmann equation description of electron transport in an electric field with cylindrical or spherical symmetry \\
\hline Author(s) & Date, H.; Shimozuma, M. \\
\hline Citation & $\begin{array}{l}\text { Physical Review E, 64(6), 066410 } \\
\text { https://doi.org/10.1103/PhysRevE.64.066410 }\end{array}$ \\
\hline Issue Date & 2001-12 \\
\hline Doc URL & http://hdl.handle.net/2115/6109 \\
\hline Rights & Copyright $\odot 2001$ American Physical Society \\
\hline Type & article \\
\hline File Information & PRE64.pdf \\
\hline
\end{tabular}

Instructions for use 


\title{
Boltzmann equation description of electron transport in an electric field with cylindrical or spherical symmetry
}

\author{
H. Date* and M. Shimozuma \\ College of Medical Technology, Hokkaido University, Sapporo 060-0812, Japan \\ (Received 26 March 2001; revised manuscript received 15 August 2001; published 26 November 2001) \\ The spatially dependent description of the electron kinetics in nonuniform electric fields is of primary \\ importance for the modeling of gas-filled proportional counters and other plasma devices. For a typical ex- \\ ample, the amplification of the gain signal in the gas counters is determined by the behavior of electron \\ ionization processes in the cylindrically or spherically symmetric electric field around thin wire or tiny sphere \\ anode. In this paper, the general formalism of the Boltzmann equation in these types of the nonuniform electric \\ fields is presented for specifying the electron swarm dynamics affected by the field geometry. The behavior of \\ electrons in the cylindrical or spherical field configuration is investigated by a Monte Carlo technique to \\ exemplify the description, and the effects associated with the angular momentum of electrons are discussed in \\ terms of the ionization coefficient.
}

DOI: 10.1103/PhysRevE.64.066410

PACS number(s): 51.50. $+\mathrm{v}, 51.10 .+\mathrm{y}, 52.80 .-\mathrm{s}$

\section{INTRODUCTION}

Applications of gas discharges to a variety of technologies such as in fabrication of new materials, etching metal surface, and neutral and ion beam sources, etc. are booming in recent years. For supporting the design and construction of the apparatus in these applications, the method of plasma modeling is being utilized with a fairly high reliability. In the modeling, there are two types of theoretical approaches: one is by the Boltzmann equation analysis for electrons, and the other is the Monte Carlo method as a numerical experiment. It goes without saying that the electron energy distribution function $(E E D F)$ is particularly essential in determining the ionization, excitation, and other processes in the weakly ionized plasmas. A complete theory with the Boltzmann equation has been established for describing the behavior of electron swarms under uniform and constant electric fields [1,2]. However, stand-alone swarm experiments in uniform and constant electric fields are of limited use. Radiation detectors and certain types of discharges are themselves swarmlike, but usually in "nonuniform" electric fields, and in-vogue types of plasma sources that one would desire to model using swarm parameters are often in a regime not well described by de swarm parameters. In recognition of this situation, the behavior of electron swarms under dynamic and/or nonuniform electric fields has begun to be characterized [3-9]. These studies have been still limited to periodically varying fields or time-harmonic fields and begin to address how swarm theory may be extended to nonlocal or nearly nonlocal conditions typical of many plasma sources $[10,11]$. There remains the need to generalize the swarm concept to electric field conditions characterized by geometries typical of those that would be found in practice. Curvature motion of electrons incorporated with the field geometry is crucial to account for the macroscopic quantity of electrons. For example, radiation detection by gas-filled counters is conducted

\footnotetext{
*Email address: date@cme.hokudai.ac.jp
}

by using cylindrical or spherical electric field conditions $[12,13]$, and a consideration of the spherical fields must be potentially important in fabrication of spherical semiconductors [14]. Moreover, inductive fields are typically curvilinear, have significant spatial gradients and come with rf magnetic fields that can be non-negligible.

In this paper, we present the Boltzmann equation description for the electron swarms under the influence of cylindrically or spherically symmetric electric fields. Typical swarm parameters, in particular the drift velocity and the ionization coefficient, in such a nonuniform electric field are investigated from a formalism with the Boltzmann equation. Next, we demonstrate a Monte Carlo simulation of electrons to specify the physical effects found in the swarm behavior in these types of nonuniform electric fields. This is a continuation of our work looking at swarm parameters, nonlocality, and scaling law. On one hand, cylindrically or spherically symmetric electric field can be the simplest model case for illustrating the behavior of electrons in nonuniform field conditions generated around fine wires, microprotrusions from material surfaces, and edges of the microtrench on semiconductor surface (which is charged up in plasma etching processes) [15]. On the other hand, cylindrically or spherically symmetric field is an excellent test for the investigation of nonlocal phenomena. As such, we present a characterization of the EEDF and swarm parameters as a function of time and location from the center of a positively biased electrode. We also discuss the general treatment of the relaxation of electron energy in the potential around the cylindrical or spherical anode.

\section{BOLTZMAN EQUATION DESCRIPTION OF THE CYLINDRICALLY AND SPHERICALLY SYMMETRIC ELECTRIC FIELDS}

The Boltzmann equation for electrons in the microvolume of six-dimensional phase space, $d \mathbf{r} d \mathbf{v}$, at time $t$ is generally expressed as 


$$
\left\{\frac{\partial}{\partial t}+\mathbf{v} \cdot \frac{\partial}{\partial \mathbf{r}}+\mathbf{a} \cdot \frac{\partial}{\partial \mathbf{v}}+J\right\} f(\mathbf{r}, \mathbf{v}, t)=0,
$$

where $\mathbf{r}$ is the position vector of electron, $\mathbf{v}$ is the velocity, $\mathbf{a}$ is the acceleration, and $J$ represents the collision operator that includes the electron-molecule collision processes [16]. For the cylindrical and spherical fields as in proportional counters, it is reasonable to transform the coordinate for the position $\mathbf{r}$ into $(r, \varphi, z)$ system for the cylindrical geometry and $(r, \theta, \varphi)$ for the spherical geometry; that is,

$$
\begin{array}{ll}
x=r \cos \varphi & \\
y=r \sin \varphi & \\
z=z & \text { for cylindrical, and } \\
x=r \sin \theta \cos \varphi & \\
y=r \sin \theta \sin \varphi & \\
z=r \cos \theta & \text { for spherical. }
\end{array}
$$

Taking account of the transformation formulas and a priori symmetry of $f(\mathbf{r}, \mathbf{v}, t)$ associated with the field geometries, we derive the Boltzmann equation in one dimension ( $r$ direction) for both the field configurations in the following subsections.

\section{A. Cylindrical geometry}

By the transformation of Eq. (1) to the cylindrical coordinate, the second term of the right-hand side of Eq. (1) can be written as,

$$
\mathbf{v} \cdot \frac{\partial f}{\partial \mathbf{r}}=v_{r} \frac{\partial f}{\partial r}+v_{\varphi} \frac{1}{r} \frac{\partial f}{\partial \varphi}+v_{z} \frac{\partial f}{\partial z}
$$

where $\left(v_{r}, v_{\varphi}, v_{z}\right)$ is the set of elements for the velocity vector $\mathbf{v}$ in this coordinate. The elements are given by

$$
\begin{gathered}
v_{r}=v_{x} \cos \varphi+v_{y} \sin \varphi, \\
v_{\varphi}=-v_{x} \sin \varphi+v_{y} \cos \varphi, \\
v_{z}=v_{z},
\end{gathered}
$$

where $\left(v_{x}, v_{y}, v_{z}\right)$ are the elements parallel to $x, y$, and $z$ axes of the velocity $\mathbf{v}$ that moves with the rotation of $\mathbf{r}$ (around the origin) keeping an angle between the velocity vector and the field line constant (see Fig. 1). Taking account of Eq. (3) and the symmetry of the function $f(\mathbf{r}, \mathbf{v}, t)$ around the anode center with respect to the velocity vector at $r$ making a fixed angle $\phi$ to the field line, we perform the integration of Eq. (2) over variables $\varphi$ and $z$. The second term in the right-hand side of Eq. (2) is replaced as

$$
v_{\varphi} \frac{1}{r} \frac{\partial f}{\partial \varphi}=\frac{1}{r}\left[-v_{x} \sin \varphi+v_{y} \cos \varphi\right] \frac{\partial f}{\partial \varphi}
$$

As the integration of each term over $\varphi$ leads to

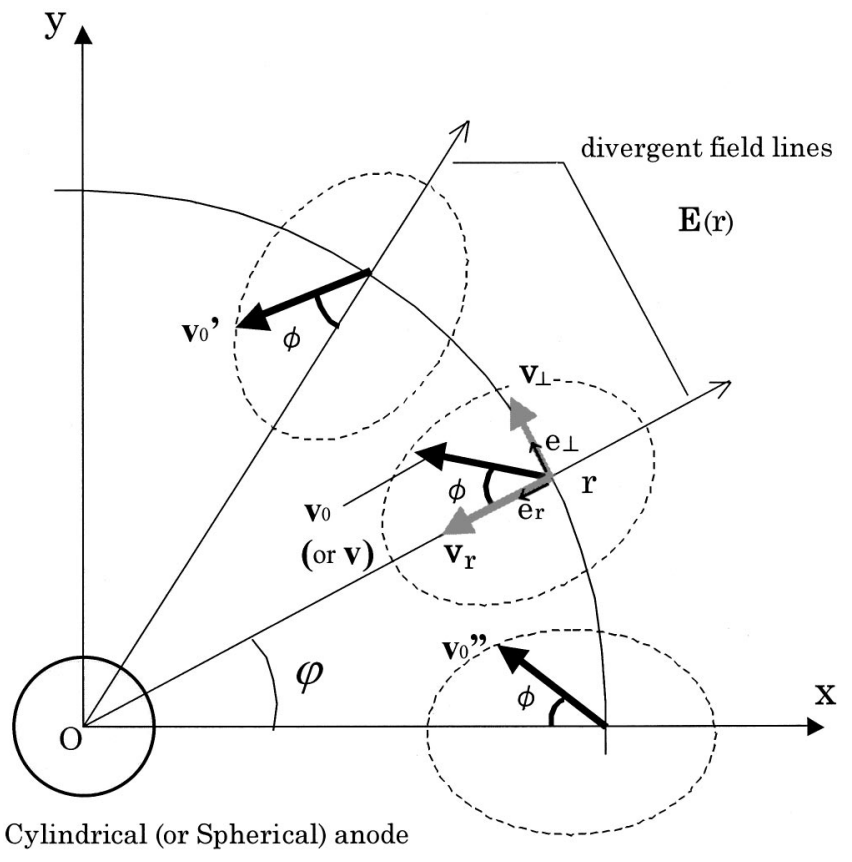

FIG. 1. Distribution symmetry of velocity vector for electron at a position $r$ in the divergent (cylindrical or spherical) electric field. The probability distribution of the vector must be symmetric with respect to the field line (for cylindrical case, this is a mirror symmetry on a plane normal to the $z$ axis). The broken-line circle represents an equiprobability surface for the distribution of velocity vector at $\mathbf{r}$. The velocity vector notation $\mathbf{v}_{0}\left(\left|\mathbf{v}_{0}\right|=\sqrt{v_{x}{ }^{2}+v_{y}^{2}}\right)$ is replaced by $\mathbf{v}\left(|\mathbf{v}|=\sqrt{v_{x}{ }^{2}+v_{y}{ }^{2}+v_{z}{ }^{2}}\right)$ for the spherical field case. If the angle $\phi$ between the velocity vector at $r$ and the field line is taken to be constant, the distribution function keeps the same value in spite of the change of angle $\varphi$ [i.e., $f\left(r, \mathbf{v}_{0}\right)=f\left(r, \mathbf{v}_{0}{ }^{\prime}\right)$ $\left.=f\left(r, \mathbf{v}_{0}{ }^{\prime \prime}\right)\right]$. The spatial integration of Eq. (1) over $\varphi$ is performed with the angle $\phi$ being unchanged. As to the acceleration term of Eq. (1), relationships below are sustained (see Appendix): $d v_{r} / d t$ $=-\left(v_{\perp}{ }^{2} / r\right)+[e E(r) / m]=\left[\left(v_{0}^{2}-v_{r}^{2}\right) / r\right]+[e E(r) / m]$ for cylindrical field and $\left[\left(v^{2}-v_{r}^{2}\right) / r\right]+[e E(r) / m]$ for spherical field.

$$
\begin{aligned}
\int_{0}^{2 \pi} \sin \varphi \frac{\partial f}{\partial \varphi} d \varphi & =[f \sin \varphi]_{0}^{2 \pi}-\int_{0}^{2 \pi} f \cos \varphi d \varphi \\
& =0-\int_{0}^{2 \pi} f \cos \varphi d \varphi
\end{aligned}
$$

and

$$
\begin{aligned}
\int_{0}^{2 \pi} \cos \varphi \frac{\partial f}{\partial \varphi} d \varphi & =[f \cos \varphi]_{0}^{2 \pi}+\int_{0}^{2 \pi} f \sin \varphi d \varphi \\
& =0+\int_{0}^{2 \pi} f \sin \varphi d \varphi
\end{aligned}
$$

we have

$$
\begin{aligned}
\frac{1}{r} \int_{0}^{2 \pi} v_{\varphi} \frac{\partial f}{\partial \varphi} d \varphi & =\frac{1}{r} \int_{0}^{2 \pi}\left[v_{x} \cos \varphi+v_{y} \sin \varphi\right] f d \varphi \\
& =\frac{1}{r} \int_{0}^{2 \pi} v_{r} f d \varphi .
\end{aligned}
$$


In regard to the third term in the right-hand side of Eq. (2), the integration over $z$ from $-\infty$ to $+\infty$ turns out to be zero as

$$
\int_{-\infty}^{+\infty} v_{z} \frac{\partial f}{\partial z} d z=\left[v_{z} f\right]_{-\infty}^{+\infty}=0 .
$$

Consequently, by the integration of Eq. (2) over spatial variables $\varphi$ and $z$, we obtain

$$
\int_{-\infty}^{+\infty} \int_{0}^{2 \pi} \mathbf{v} \cdot \frac{\partial f(r, \varphi, z)}{d \mathbf{r}} d \varphi d z=2 \pi\left[v_{r}\left(\frac{\partial}{\partial r}+\frac{1}{r}\right) f(r, \mathbf{v}, t)\right] .
$$

Here, it should be emphasized that the integration above has been made by taking advantage of the symmetry of the function for the velocity vector at a certain angle to the field line as shown in Fig. 1.

Next, we take the third term of Eq. (1) into account. The acceleration $\mathbf{a}=d \mathbf{v} / d t$ is seemingly given by $[e E(r)] / m$ along $r$ direction [ $e$ and $m$ are the charge and mass of electron, and $E(r)$ is the $r$-dependent electric field]. However, the motion of electron orbiting around the anode wire with the conservation of angular momentum is not to be considered by this expression. In order to incorporate this angular effect into the Boltzmann equation description, the "normal acceleration" term induced by the velocity element $\left(v_{\varphi}\right)$ normal to the field direction should be added as follows.

$$
\frac{d v_{r}}{d t}=-\frac{v_{\varphi}^{2}}{r}+\frac{e E(r)}{m}=-\frac{\left(v_{0}^{2}-v_{r}^{2}\right)}{r}+\frac{e E(r)}{m},
$$

where $v_{0}^{2}=v^{2}-v_{z}^{2}$ (see Appendix). It should be noted that the vector element in $r$ direction is set to be positive toward the anode. Then the integration of $(d \mathbf{v} / d t)(\partial f / \partial \mathbf{v})$ over $d \varphi d z$ gives us

$$
\begin{aligned}
\int_{-\infty}^{+\infty} \int_{0}^{2 \pi} \frac{d \mathbf{v}}{d t} \frac{\partial f}{\partial \mathbf{v}} d \varphi d z= & 2 \pi\left[-\frac{\left(v_{0}^{2}-v_{r}^{2}\right)}{r}\right. \\
& \left.+\frac{e E(r)}{m}\right] \frac{\partial f(r, \mathbf{v}, t)}{\partial v_{r}} .
\end{aligned}
$$

Finally, as the results of the integration over spatial variables except $\mathbf{r}$, the equation for $F(r, \mathbf{v}, t)[\equiv r f(r, \mathbf{v}, t)$ $\left.=(1 / 2) \pi \iint f(\mathbf{r}, \mathbf{v}, t) r d \varphi d z\right]$ is deduced as,

$$
\left\{\frac{\partial}{\partial t}+v_{r} \frac{\partial}{\partial r}+\left[-\frac{\left(v_{0}^{2}-v_{r}^{2}\right)}{r}+\frac{e E(r)}{m}\right] \frac{\partial}{\partial v_{r}}+J\right\} F(r, \mathbf{v}, t)=0 .
$$

\section{B. Spherical geometry}

In a manner similar to the cylindrical case, the integration over $\theta$ and $\varphi$ can be made based upon $d \mathbf{r}=r^{2} \sin \theta d \theta d \varphi d r$ for spherical coordinate. The second term in the left-hand side of Eq. (1) is

$$
\mathbf{v} \cdot \frac{\partial f}{\partial \mathbf{r}}=v_{r} \frac{\partial f}{\partial r}+v_{\theta} \frac{1}{r} \frac{\partial f}{\partial \theta}+v_{\varphi} \frac{1}{r \sin \theta} \frac{\partial f}{\partial \varphi},
$$

and integrating this by $d \Omega=\sin \theta d \theta d \varphi$, we have

$$
\int \mathbf{v} \cdot \frac{\partial f}{\partial \mathbf{r}} d \Omega=4 \pi\left[v_{r}\left(\frac{\partial}{\partial r}+\frac{2}{r}\right) f(r, \mathbf{v}, t)\right] .
$$

As to the third term in the left-hand side of Eq. (1), the acceleration can be described for the spherical model as (see Appendix),

$$
\frac{d v_{r}}{d t}=-\frac{v_{\perp}^{2}}{r}+\frac{e E(r)}{m}
$$

where $v_{\perp}^{2}=v^{2}-v_{r}{ }^{2}$. Performing the integration of $(d \mathbf{v} /$ $d t)(\partial f / \partial \mathbf{v})$ over $d \Omega$ leads to

$$
\int \frac{d \mathbf{v}}{d t} \frac{\partial f}{\partial \mathbf{v}} d \Omega=4 \pi\left[-\frac{\left(v^{2}-v_{r}^{2}\right)}{r}+\frac{e E(r)}{m}\right] \frac{\partial f(r, \mathbf{v}, t)}{\partial v_{r}} .
$$

Thus, the final form of the one-dimensional Boltzmann equation for spherical fields is expressed by

$$
\left\{\frac{\partial}{\partial t}+v_{r} \frac{\partial}{\partial r}+\left[-\frac{\left(v^{2}-v_{r}^{2}\right)}{r}+\frac{e E(r)}{m}\right] \frac{\partial}{\partial v_{r}}+J\right\} F(r, \mathbf{v}, t)=0,
$$

where

$$
F(r, \mathbf{v}, t) \equiv r^{2} f(r, \mathbf{v}, t)=[1 / 4 \pi] \iint f(r, \mathbf{v}, t) r^{2} \sin \theta d \theta d \varphi .
$$

\section{DERIVATION OF THE IONIZATION COEFFICIENT}

In this section, using Eqs. (9) and (14) for cylindrical and spherical field geometries, we deduce the expression of the ionization coefficient that is essential in evaluating the electron multiplication factor in gas counters. The ionization coefficient was defined originally as the multiplication rate of electron number per unit length along the field direction by Townsend at the turn of the 20th century. On this coefficient we have discussed from a view point of the arrival-time spectra (ATS) method in a previous paper [17]. In the ATS method, the ionization coefficient is regarded as the lowest order parameter of the time derivative expansion of $N(r, t)$ and is given by $[1 / N(r)][\partial N(r) / \partial r]$. Here, $N(r, t)$ $=\int F(r, \mathbf{v}, t) d \mathbf{v}$ and $N(r)=\int N(r, t) d t$. However, as mentioned in the paper, this definition is not appropriate to describe the electron multiplication factor in nonuniform electric fields such as in the proportional counters. In order to capture the number of electrons passing through a plane (normal to the field line) at an arbitrary position $r$, we have to consider the electron flux, $\Gamma(r, t) \equiv V_{d}(r, t) N(r, t)\left[V_{d}(r, t)\right.$ is the drift velocity toward the anode], not the electron number density $N(r, t)$ that observed in $d r$ at $t$; then the total number of electrons is obtained by integrating $\Gamma(r, t)$ over entire time $t$. Ultimately, the ionization coefficient as a function of position $r$ must be physically defined by

$$
\alpha(r)=\frac{1}{\Gamma(r)} \frac{\partial \Gamma(r)}{\partial r} \quad\left[\Gamma(r) \equiv \int V_{d}(r, t) N(r, t) d t\right] .
$$


However, it is worthy of note that $\alpha(r)$ defined above is equivalent to that given by $[1 / N(r)][\partial N(r) / \partial r]$ if the "uniform" field condition with a hydrodynamic regime [1] is the case.

To proceed to the deduction of $\alpha(r)$ from Eq. (9) or Eq. (14), the distribution function $F(r, \mathbf{v}, t)$ shall be written as $F(r, \mathbf{v}, t)=g(r, \mathbf{v}, t) N(r, t)$, where $g(r, \mathbf{v}, t)$ is the normalized velocity distribution function (i.e., $\int g(r, \mathbf{v}, t) d \mathbf{v}=1$ ). According to this expression, Eq. (9) is rewritten by

$$
\begin{aligned}
\frac{\partial g}{\partial t} N & +g \frac{\partial N}{\partial t}+v_{r}\left(\frac{\partial g}{\partial r} N+g \frac{\partial N}{\partial r}\right)+\left[-\frac{\left(v_{0}^{2}-v_{r}^{2}\right)}{r}\right. \\
+ & \left.\frac{e E(r)}{m}\right] \frac{\partial g}{\partial v_{r}} N+J(g N)=0 .
\end{aligned}
$$

Taking the symmetry of velocity space associated with the field geometries into account, the operator $\partial / \partial v_{r}$ is given by

$$
\frac{\partial}{\partial v_{r}}=\cos \phi \frac{\partial}{\partial v_{0}}+\frac{\sin ^{2} \phi}{v_{0}} \frac{\partial}{\partial[\cos \phi]},
$$

where $v_{r}=v_{0} \cos \phi$ and $\phi$ is the polar angle in the velocity space as shown in Fig. 1. By using this formula, the integration over $d \mathbf{v}=v_{0} d \phi d v_{0} d v_{z}$ is performed as

$$
\int v_{0}^{2} \frac{\partial g(r, \mathbf{v})}{\partial v_{r}} d \mathbf{v}=-2 \int v_{r} g(r, \mathbf{v}) d \mathbf{v} .
$$

At the same time,

$$
\int v_{r}{ }^{2}\left[\partial g(r, \mathbf{v}) / \partial v_{r}\right] d \mathbf{v}=-2 \int v_{r} g(r, \mathbf{v}) d \mathbf{v}
$$

holds as well, and then the integration over $d \mathbf{v}$ with respect to the third term in Eq. (9) turns out to be zero. Therefore, we have the continuity equation of electron number in a wellknown form as,

$$
\frac{\partial N(r, t)}{\partial t}+\frac{\partial}{\partial r}\left[V_{d}(r, t) N(r, t)\right]=R_{i a}(r, t) N(r, t) .
$$

Here, $R_{i a}(r, t)$ is the effective ionization frequency [i.e., $R_{i a}(r, t) \equiv R_{i}(r, t)-R_{a}(r, t) ; R_{i}(r, t)$ is the ionization frequency at $r$ and $t$, and $R_{a}(r, t)$ is the attachment frequency]. By the integration of Eq. (19) over time $t$ from zero to infinity, the first term of the left-hand side of Eq. (19) vanishes in an effective drift region of electrons. Here, the effective drift region means the discharge space between the starting point of the initial electrons and the anode surface. Finally, considering the definition of the ionization coefficient in Eq. (15), we obtain

$$
\alpha(r)=\frac{R_{i a}(r)}{V_{d}(r)}=\frac{N_{g} \int v q_{i a}(v) g(r, \mathbf{v}) d \mathbf{v}}{\int v_{r} g(r, \mathbf{v}) d \mathbf{v}},
$$

where $N_{g}$ is the number density of background gas molecules, $q_{i a}$ is $q_{i}-q_{a}\left(q_{i}\right.$ and $q_{a}$ are ionization and attachment cross sections). Also for the spherical field model, the deduction of the equations same as Eqs. (16)-(20) can be made

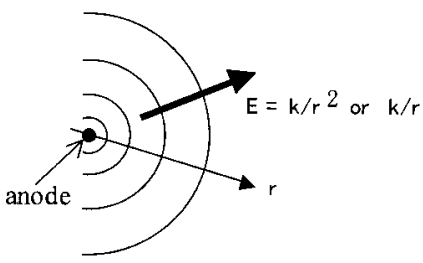

(a) spherical or cylindrical

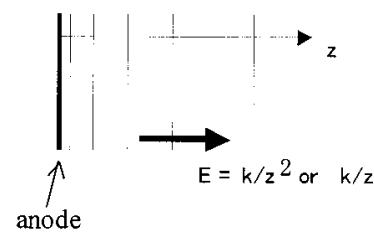

(b) parallel plane
FIG. 2. Field geometry models: (a) for spherical or cylindrical (divergent) configuration, and (b) for parallel plane.

with the replacement of $v_{0}$ by $v$. It should be noted that the formalism of the continuity equation of electron number and the ionization coefficient, in $r$ direction, in the cylindrical or spherical field geometry is completely identical to that in the parallel (nondivergent) field condition, even though the original equation before the integration [i.e., Eq. (9) or Eq. (14)] is different from the common Boltzmann equation for onedimensional parallel fields.

\section{MODEL OF A MONTE CARLO METHOD}

In Sec. II, we have deduced the one-dimensional Boltzmann equation of electrons in the electric field with cylindrical or spherical symmetry [i.e., Eqs. (9) and (14)]. Unfortunately, it is difficult to solve the differential equations (9) and (14) by any numerical means since these equations depend on both velocity vector $\mathbf{v}$ and position $r$. However, it must be important to know the Boltzmann equation for these types of field geometry because the conventional analyses have substituted a simple one-dimensional Boltzmann equation (ignoring the effects by the divergent electric fields) for the "intrinsic" expression of the equation. Disregarding the effects associated with the field geometry may cause an inconsistency in the methodology to describe the macroscopic electron behavior. As a typical example, the ionization coefficient has been deduced properly from Eqs. (9) and (14) in the preceding Sec. III. Specifically, the deduction also includes the consideration of volumetric factors $r$ and $r^{2}$.

In order to illustrate the kinetics of electrons in the nonuniform electric fields and to confirm the validity of Eqs. (15) and (20), we perform a Monte Carlo simulation as a numerical experiment. In this simulation, the effects of the orbiting motion of electrons (with the angular momentum) on the transport parameters are the focus of attention. Figure 2 shows the field models used in this study. The electron dynamics in the cylindrical or spherical field model [Fig. 2 (a) ] is compared with that in the parallel plane geometry having the same spatial dependence of field strength along the field line [Fig. 2(b)], aiming to see the effects of the angular momentum of electrons in the "divergent" electric fields. Initial electrons are released at a certain distance from the anode, and the motion of them involving the collision events with background gas molecules and the drift toward the anode is followed up to the anode surface by a Monte Carlo technique.

Table I shows the condition of the simulation. The Monte Carlo technique used here is based upon the free-flight-time method [18], in which the time step for the technique is set 
TABLE I. Simulation condition.

\begin{tabular}{ll}
\hline \hline Field model & \\
Geometry & $k / r$ or $k / r^{2}\left(k / z\right.$ or $\left.k / z^{2}\right)$ \\
Radius of cylindrical or spherical anode $\left(r_{0}\right)$ & $0.1-0.5 \mathrm{~mm}$ \\
Gas & Ar-like and $\mathrm{Cl}_{2}$-like models \\
Anode voltage & $500-900 \mathrm{~V}$ \\
Gas pressure & $1.0 \mathrm{Torr}$ \\
Condition for the initial electrons & \\
Number of the electrons & $20000-60000$ \\
Released position & 9.9 or $9.5 \mathrm{~mm}$ from the anode surface \\
Direction & at random \\
Energy & Maxwellian distribution with a mean \\
& energy ranging from 0.1 to $10 \mathrm{eV}$ \\
Parameters for the Monte Carlo simulation $($ free-flight-time mehtod $[18])$ \\
Time step $(\Delta t)$ & $<0.5 \mathrm{psec}$ \\
Simulation time & $50-200 \mathrm{nsec}$ \\
Bin's width for spatial sampling $(\Delta r$ or $\Delta z)$ & $0.1 \mathrm{~mm}$ \\
\hline \hline
\end{tabular}

small enough to trace the electron trajectory accurately $(\Delta t$ is typically less than $10^{-12} \mathrm{sec}$ ). The electrons see a cylindrical or spherical electrode that is positively biased and its center is located at the origin with radius $0.1-0.5 \mathrm{~mm}$. Initial electrons are released at a point on a shell about the anode (typically $1.0 \mathrm{~cm}$ point from the anode center) with a Maxwellian distribution at a mean energy of $0.1-10 \mathrm{eV}$, and the single particle simulation (including the second electron generation) is iterated for a number of the initial electrons. To make comparison with the cylindrical or spherical model analysis, simulations in parallel plane fields with the same field profile along the field line (corresponding to the respective model) are carried out as well. Field distortion by the space charge is assumed to be negligible. Sampling is made using a large number of test electrons (greater than 20000), which are followed for several hundreds of nanoseconds. We can ignore the motion of the background gas molecules compared with that of electrons. The positive bias voltage is varied from 500 to $900 \mathrm{~V}$ and it is assumed that the anode absorbes arriving electrons without reflection. Secondary electron generation at the cathode through photons and/or ions is not included.

Two types of model gases, a $\mathrm{Cl}_{2}$-like model and an Ar-like model, are the focus of the simulation. A set of cross sections for the $\mathrm{Cl}_{2}$-like model is same as is shown in a previous paper [19]. The total cross section of this model is set to be a constant value of $10^{-15} \mathrm{~cm}^{2}$ for generality, there is no impact otherwise from this assumption. A set of cross sections for the Ar-like model is given by the following functions:

$q_{i}($ ionization $)$ :

$$
\begin{aligned}
& \left(0.5 \times 10^{-16}\right) \sqrt{\varepsilon-15.76} \\
& \times \exp \left(-\frac{\varepsilon}{200}\right) \mathrm{cm}^{2} \text { for } \varepsilon \geqslant 15.76 \mathrm{eV},
\end{aligned}
$$

$$
\begin{aligned}
& q_{\mathrm{ex}}(\text { excitation }): \\
& \quad\left(0.3 \times 10^{-16}\right) \sqrt{\varepsilon-11.55} \\
& \quad \times \exp \left(-\frac{\varepsilon}{150}\right) \mathrm{cm}^{2} \text { for } \varepsilon \geqslant 11.55 \mathrm{eV},
\end{aligned}
$$

$q_{m}$ (momentum transfer):

$$
\begin{aligned}
& \left(1.0 \times 10^{-15}\right)[(1.0-3.66) \sqrt{\varepsilon-0.001} \\
& \left.\quad \times \exp \left(-\frac{\varepsilon}{0.4}\right)\right] \mathrm{cm}^{2} \text { for } \varepsilon<0.2 \mathrm{eV}, \\
& \left(1.0 \times 10^{-15}\right)\left(\frac{10}{\varepsilon}\right)^{-1.177184} \mathrm{~cm}^{2} \text { for } 0.2 \mathrm{eV} \leqslant \varepsilon<10 \mathrm{eV}, \\
& \left(1.0 \times 10^{-15}\right)\left(\frac{10}{\varepsilon}\right)^{0.7} \mathrm{~cm}^{2} \text { for } 10 \mathrm{eV} \leqslant \varepsilon,
\end{aligned}
$$

where the ionization and the excitation threshold energies are chosen to be 15.76 and $11.55 \mathrm{eV}$, respectively.

\section{SIMULATION RESULTS AND DISCUSSION}

In Fig. 3(a), we show a set of typical snapshots of spatially resolved electron number for the spherical field condition. The total number of electrons over the spherical shell volume, which can be obtained by integrating the distribution over $r$ (from the anode surface position $r_{0}$ to $\infty$ ) at each sampling time in Fig. 3(a), increases for a time and then decreases monotonically owing to the absorption of electrons into the anode. A remarkable feature of the electron number distribution is the lack of variation of the spatial profile after several tens of nanoseconds. This suggests that the electrons might be in a steady state or, namely, a hydrodynamic equilibrium state, keeping the same spatial distribution after a 


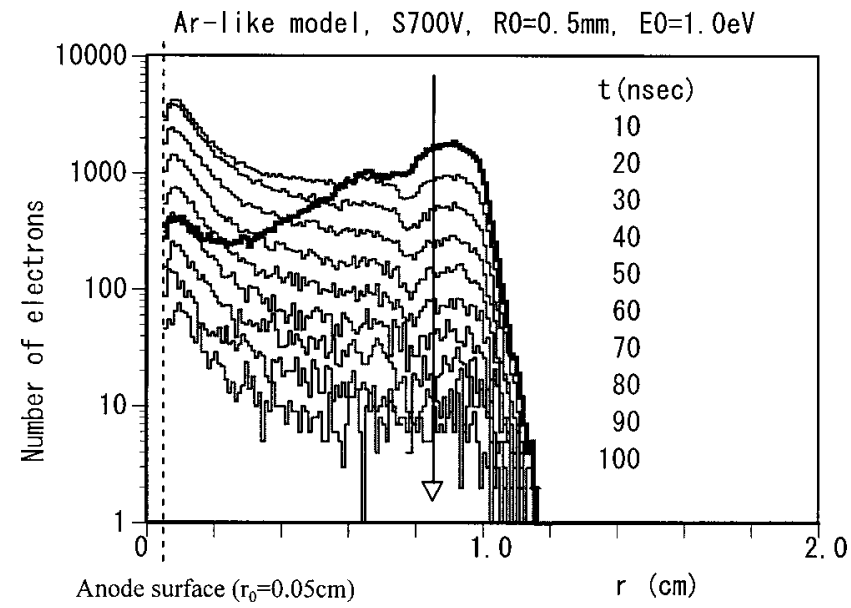

(a)

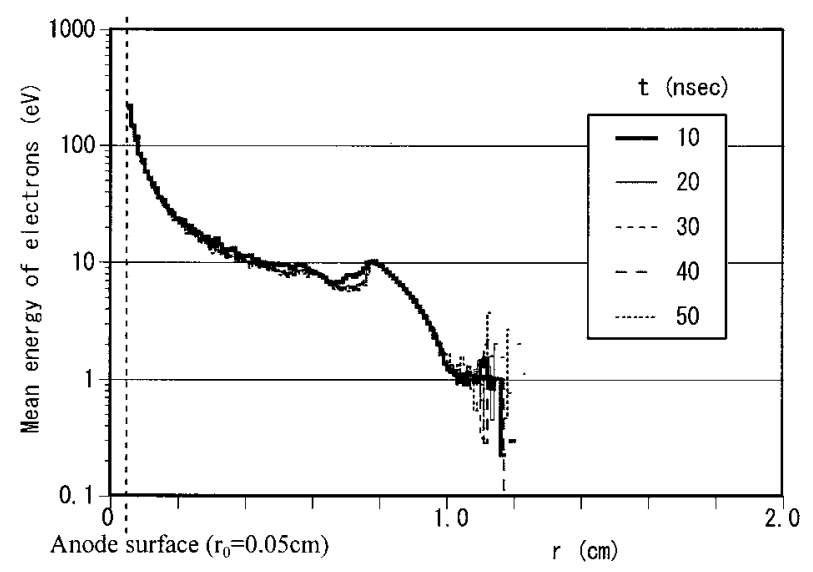

(b)

FIG. 3. Spatially resolved quantities for Ar-like model gas at $700-\mathrm{V}$ bias voltage of spherical anode ( $0.5 \mathrm{~mm}$ radius). (a) for electron number and (b) for mean energy of electrons. Initial electrons with a Maxwellian distribution (at $1.0 \mathrm{eV}$ mean energy) were released at $r=1.0 \mathrm{~cm}$.

certain time. Spatial distribution of the mean energy of electrons in Fig. 3(b) shows evidence of the steady-state phase of electrons. These characteristics were recognized for a variety of initial conditions of electron energy from 0.1 to $10 \mathrm{eV}$ and also for the cylindrical and parallel field [17] configurations. The entire results imply that the electron transport parameters are in an equilibrium state, being uniquely determined by the local field strength, after a relatively short period of time under this type of nonuniform field condition.

Figure 4 shows the comparison between the parameters in cylindrical and parallel field models. One-dimensional field strength is varied equally for both the models. The drift velocity $\left[V_{d}(r)\right.$ or $\left.V_{d}(z)\right]$ and the ionization frequency $\left[R_{i}(r)\right.$ or $\left.R_{i}(z)\right]$ were calculated by accumulating $v_{r}\left[=v_{0} \cos \phi\right]$ and $v q_{i}(v)$ for all electrons at each location and by averaging them per electron over entire time. The ionization coefficient was given by $\alpha=R_{i} / V_{d}$ following the definition of Eq. (20). Here, $R_{i}$ is identical to $R_{i a}$ for Ar-like gas because there is no attachment collision process, and the entire time means the period from $t=0$ up to the time when the rear end electron in the swarm is absorbed into the anode. The differ-

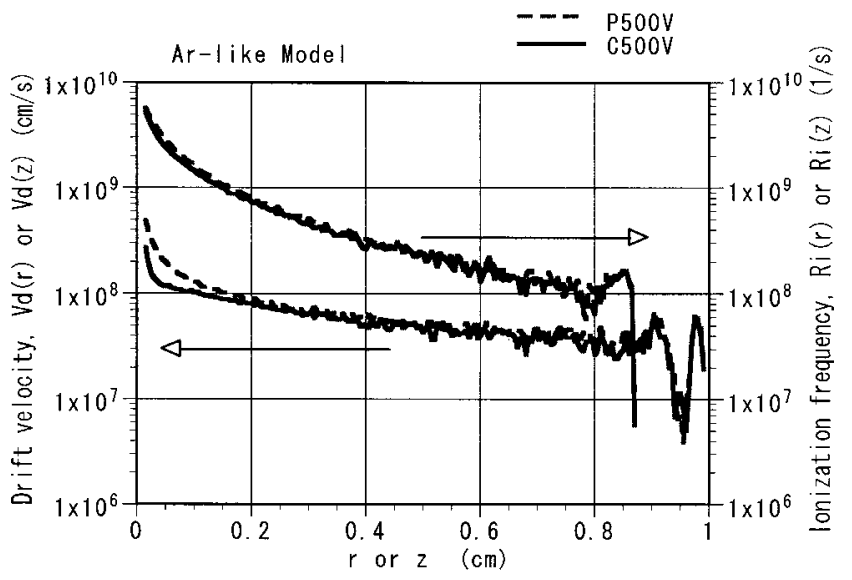

(a)

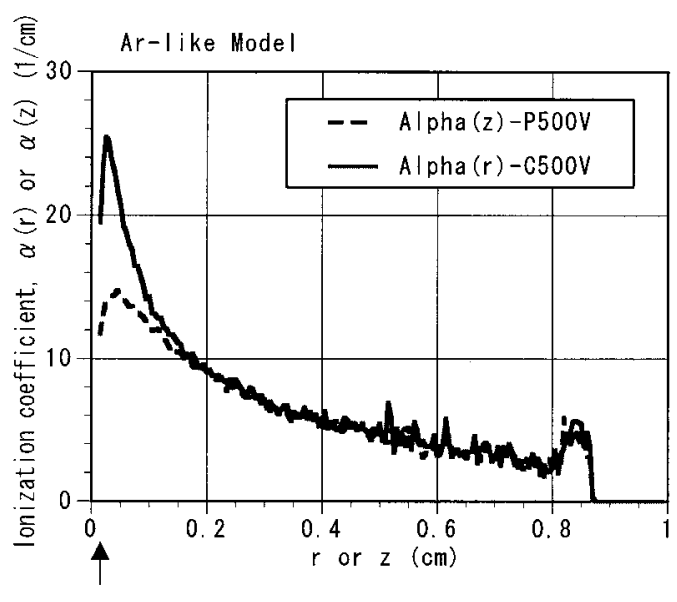

(b)

FIG. 4. Comparison of the parameters in cylindrical and parallel field models: (a) for the drift velocity $V_{d}$ and the ionization frequency $R_{i}$, and (b) for the ionization coefficient $\alpha=R_{i} / V_{d}$. Radius of cylindrical anode is $0.1 \mathrm{~mm}$, and the anode voltage for both models is $500 \mathrm{~V}$. Initial electrons start at $1.0 \mathrm{~cm}$ with a Maxwellian distribution at $0.1 \mathrm{eV}$ mean energy.

ence between the parameters for both field models represents the effect of electron motion with angular momentum around the anode, which may be described by the additional term with $-\left(v_{0}^{2}-v_{r}^{2}\right) / r$ in the left-hand side of Eq. (9). It is noted that the ionization coefficient in the cylindrical field is much greater than that in the parallel field in the vicinity of the anode surface. This gap is mainly caused by the difference between the drift velocities in both the models. In the cylindrical field geometry, the drift velocity of electrons along the field line is reduced effectively by the spiral motion of them around the anode. Similarly to Fig. 4, we present the comparison of the parameters in spherical and parallel models for $\mathrm{Cl}_{2}$-like gas in Fig. 5. The same effect on the ionization coefficient is recognized in Fig. 5(b).

As is well known in the study on gas-filled radiation detectors [20], the ionization coefficient leads to the derivation of the gas gain or the multiplication factor of electrons in proportional counters as 


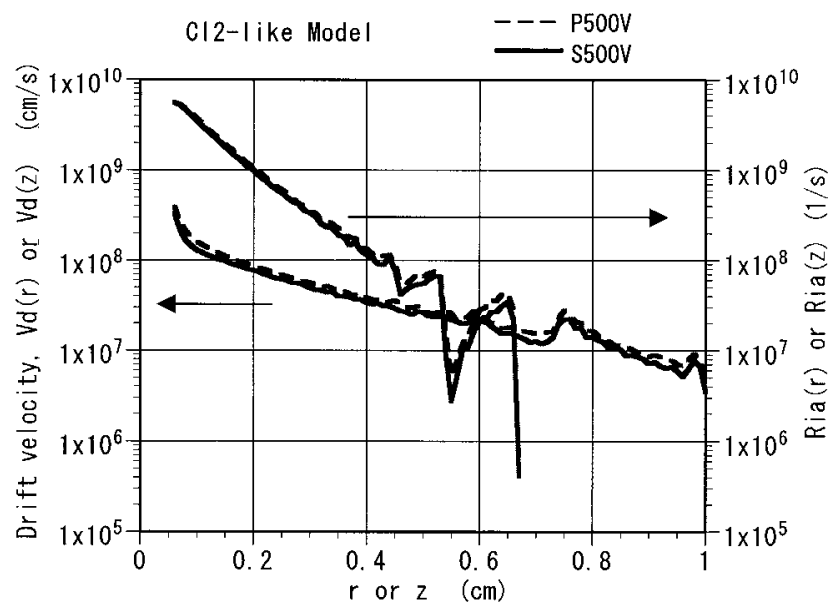

(a)

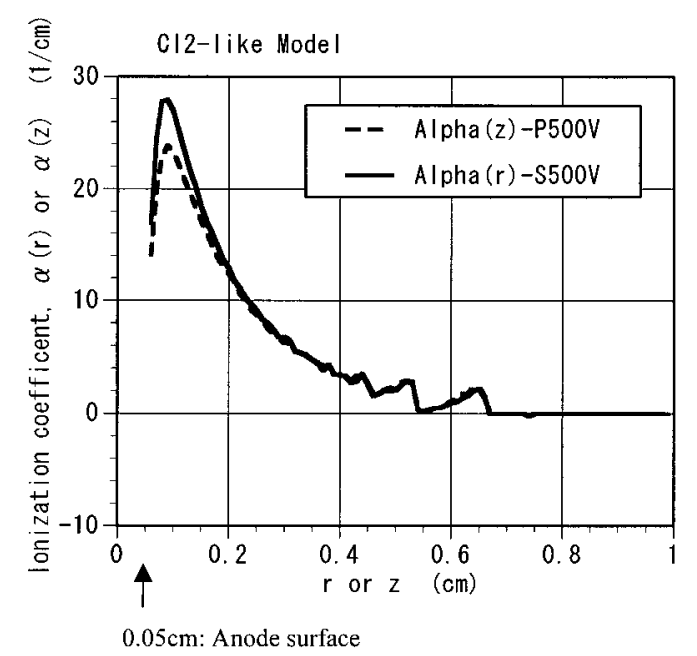

(b)

FIG. 5. Comparison of the parameters in spherical and parallel field models: (a) for the drift velocity $V_{d}$ and the effective ionization frequency $R_{i a}$, and (b) for the ionization coefficient $\alpha$ $=R_{i a} / V_{d}$. Radius of spherical anode is $0.5 \mathrm{~mm}$, and the anode voltage for both models is $500 \mathrm{~V}$. Initial electrons start at $1.0 \mathrm{~cm}$ with a Maxwellian distribution at $0.1 \mathrm{eV}$ mean energy.

$$
\ln G=\int_{A}^{B} \alpha(r) d r
$$

where $A$ and $B$ represent the boundary of discharge region in the field. If the minimum boundary $A$ is set to be the anode radius, the gain $G$ becomes equivalent to the total number of electrons arrived at the anode surface arising from an initial electron at a position $B$ far from the anode. In Table II, the mean value of the gain (per initial electron) is listed for various conditions, and we can see that the field of cylindrical or spherical geometry enhances the gain significantly. It is noteworthy that the electron motion with the angular momentum encourages the energy transfer (through the collision events) to the background gases.

\section{SUMMARY AND CONCLUSIONS}

In this study, we have shown the Boltzmann equation pertinent to describe the electron transport in the electric fields with cylindrical or spherical symmetry. Then, the typical transport parameter, the ionization coefficient, has been investigated in connection with the multiplication factor of gas counters. In order to illustrate the physical effects implicated in the Boltzmann equation, we have demonstrated a Monte Carlo simulation of electrons in the divergent electric fields.

The present study leads to the following conclusions:

(1) A modification of the acceleration term in the Boltzmann equation (one-dimensional in space) is necessary for describing electron swarms in the field with cylindrical or spherical symmetry. The additional acceleration term arises from the velocity element of electrons normal to the field direction. The Monte Carlo simulation results support the Boltzmann equation description.

(2) The ionization coefficient derived from the onedimensional Boltzmann equation for the cylindrical or spherical field has an identical form to that in nondivergent field geometry, in spite of the difference in the original equation. In addition, it should be noted that the coefficient for evaluating the multiplication factor of electron number in the nonuniform electric fields is correctly given by the flux consideration of electrons, not by the spatial electron density.

(3) The electron swarm behavior involving the collision processes with background gases and the drift motion is

TABLE II. Comparison of the gain.

\begin{tabular}{lll}
\hline \hline (Ar-like gas model $)$ & & \\
Cylindrical $\left(r_{0}=0.5 \mathrm{~mm}\right)$ & Parallel plane & $V_{a}=500 \mathrm{~V}$ \\
$G=463.4$ & $G=244.1$ & $V_{a}=500 \mathrm{~V}$ \\
Cylindrical $\left(r_{0}=0.1 \mathrm{~mm}\right)$ & Parallel Plane & \\
$G=1098.9$ & $G=874.7$ & $V_{a}=500 \mathrm{~V}$ \\
Spherical $\left(r_{0}=0.5 \mathrm{~mm}\right)$ & Parallel plane & $V_{a}=700 \mathrm{~V}$ \\
$G=40.9$ & $G=29.8$ & \\
$G=94.8$ & $G=65.0$ & $V_{a}=500 \mathrm{~V}$ \\
$\left(\mathrm{Cl}_{2}\right.$-like gas model $)$ & & $V_{a}=900 \mathrm{~V}$ \\
Spherical $\left(r_{0}=0.5 \mathrm{~mm}\right)$ & Parallel plane & $G=93.4$ \\
$G=131.0$ & $G=676.4$ & \\
$G=1218.7$ & & \\
\hline \hline
\end{tabular}


strongly influenced by the angular momentum of electrons near the source of divergent electric fields. It is suggested that the local equilibrium of EEDF under the $1 / r$ or $1 / r^{2}$ type of field geometry can be led in a short period of time.

As an extension, it is possible to infer that the effects of angular momentum of electrons may be added into the ac celeration term in the Boltzmann equation of electrons also for some types of magnetic field conditions.

\section{ACKNOWLEDGMENTS}

The authors are indebted to Professor H. Tagashira, Professor T. Yamamoto, Professor K. Kitamori, Professor K. Kondo, and to Dr. P. L. G. Ventzek for valuable discussions.

\section{APPENDIX}

As to the third term of the left-hand side of Eq. (1), we can transform it to the expression in cylindrical or spherical coordinates as follows:

$$
\begin{aligned}
\frac{d \mathbf{v}}{d t} \cdot \frac{\partial f}{\partial \mathbf{v}} & =\frac{d v_{x}}{d t} \frac{\partial f}{\partial v_{x}}+\frac{d v_{y}}{d t} \frac{\partial f}{\partial v_{y}}+\frac{d v_{z}}{d t} \frac{\partial f}{\partial v_{z}} \\
& =\frac{d v_{r}}{d t} \frac{\partial f}{\partial v_{r}}+\frac{d v_{\varphi}}{d t} \frac{\partial f}{\partial v_{\varphi}}+\frac{d v_{z}}{d t} \frac{\partial f}{\partial v_{z}}
\end{aligned}
$$

for the cylindrical coordinates, and

$$
=\frac{d v_{r}}{d t} \frac{\partial f}{\partial v_{r}}+\frac{d v_{\theta}}{d t} \frac{\partial f}{\partial v_{\theta}}+\frac{d v_{\varphi}}{d t} \frac{\partial f}{\partial v_{\varphi}}
$$

for the spherical coordinates.

According to the Newton's second law of motion in a cylindrical and a spherical field potential, the acceleration in the $r$ direction is given by

$$
a_{r}=\ddot{r}-r \dot{\varphi}^{2}=-\frac{\partial V(r, \varphi, z)}{\partial r}
$$

for cylindrical and

$$
a_{r}=\ddot{r}-r\left[\dot{\theta}^{2}+\dot{\varphi}^{2} \sin ^{2} \theta\right]=-\frac{\partial V(r, \theta, \varphi)}{\partial r}
$$

for spherical fields, respectively. Then, letting the velocity element toward the origin be positive in the radial direction, we obtain

$$
\frac{d v_{r}}{d t}=\ddot{r}=-\frac{v_{\varphi}^{2}}{r}+\frac{e E(r)}{m}
$$

for cylindrical and

$$
\frac{d v_{r}}{d t}=\ddot{r}=-\frac{v_{\theta}^{2}+v_{\varphi}^{2}}{r}+\frac{e E(r)}{m}
$$

for spherical configurations.

Other terms associated with $d v_{\varphi} / d t$ and $d v_{z} / d t$ or $d v_{\varphi} / d t$ and $d v_{\theta} / d t$ can be eliminated by the integration over the spatial variables except $r$ owing to the symmetry of the function $f$ in velocity space. Here, it should be noted that the integration is carried out keeping the velocity vector to make a same angle with respect to the field line (see Fig. 1).
[1] K. Kumar, H. R. Skullerud, and R. E. Robson, Aust. J. Phys. 33, 343 (1980).

[2] K. Kondo and H. Tagashira, J. Phys. D 23, 1175 (1990).

[3] T. J. Moratz, L. C. Pitchford, and J. N. Bardsley, J. Appl. Phys. 61, 2146 (1987).

[4] M. Matoba, T. Hirose, T. Sakae, H. Kametani, H. Ijiri, and T. Shintake, IEEE Trans. Nucl. Sci. NS-32, 541 (1985).

[5] M. A. Lieberman, J. Appl. Phys. 65, 4186 (1988).

[6] N. L. Aleksandrov and I. V. Kochetov, J. Phys. D 29, 1476 (1996).

[7] H. Date, P. L. G. Ventzek, M. Shimozuma, and H. Tagashira, J. Appl. Phys. 79, 2902 (1996).

[8] F. Sigeneger and R. Winkler, Plasma Chem. Plasma Process. 17, 11 (1997).

[9] R. D. White, R. E. Robson, and K. F. Ness, J. Vac. Sci. Technol. A 16, 316 (1998).

[10] V. I. Kolobov, D. F. Beale, L. J. Mahoney, and A. E. Wendt, Appl. Phys. Lett. 65, 37 (1994).

[11] U. Kortshagen, I. Purkropski, and L. D. Tsendin, Phys. Rev. E
51, 6063 (1995).

[12] P. Ségur, I. Pérès, J. P. Boeuf, and J. Barthe, Radiat. Prot. Dosim. 31, 107 (1990).

[13] P. Ségur, P. Olko, and P. Colautti, Radiat. Prot. Dosim. 61, 323 (1995).

[14] A. Ishikawa, in Proceedings of 1998 International Conference on Solid State Devices and Materials A (Business Center for Academic Societies, Tokyo, Japan 1998), Vol. 1, p. 428.

[15] G. S. Hwang and K. P. Giapis, J. Appl. Phys. 84, 154 (1998).

[16] H. Date, K. Kondo, and H. Tagashira, J. Phys. D 23, 1384 (1990).

[17] H. Date, N. Ikuta, K. Kondo, K. Sato, M. Shimozuma, and H. Tagashira, Jpn. J. Appl. Phys., Part 1 39, 6043 (2000).

[18] H. Tagashira, ed., Technical Report (Pt. II) No. 140, IEE, Tokyo, Japan (1982) (in Japanese).

[19] H. Date, P. L. G. Ventzek, K. Kondo, H. Hasegawa, M. Shimozuma, and H. Tagashira, J. Appl. Phys. 83, 4024 (1998).

[20] T. Aoyama, Nucl. Instrum. Methods Phys. Res. A 234, 125 (1985). 